Wolf Strecker ${ }^{1} \cdot$ Peter Keppler ${ }^{2} \cdot$ Michael Schulte $^{3}$

${ }^{1}$ Klinik für Unfall-, Hand- und Wiederherstellungschirurgie, Klinikum Bamberg

${ }^{2}$ Klinik für Unfall-, Hand- und Wiederherstellungschirurgie, Chirurgische Universitätsklinik Ulm

${ }^{3}$ Unfall- und Wiederherstellungschirurgie, Diakoniekrankenhaus Rotenburg/Wümme

\title{
Maßnahmen bei fehlgeschlagenen Osteosynthesen am proximalen Femur
}

$$
\text { Epidemiologie }
$$

Operative Versorgungen proximaler $\mathrm{Fe}$ murfrakturen sind nicht frei von Problemen und Fehlschlägen. Deren Ursachen und Häufigkeiten werden von verschiedenen Autoren in dem vorliegenden Supplementsband von „Trauma und Berufskrankheit“ ausführlich dargestellt. Hierbei werden altersbezogene Konstellationen ebenso berücksichtigt wie verschiedene Konzepte der osteosynthetischen $[1,5,6,18]$ und endoprothetischen [8] Versorgung.

Fehlgeschlagene Osteosynthesen lassen sich bezüglich Ursachen und Auswirkungen in folgende Schwerpunkte zusammenfassen:

- implantatassoziierte Probleme

- Störungen der Knochenheilung

- Deformitäten

- Infekte

\section{Implantatassoziierte Probleme} bildgebende Verfahren. Entsprechende Indikationen zur Korrektur ein- und mehrdimensionaler Deformitäten werden ebenso angegeben wie Beispiele der operativen Umsetzung. Die Behandlung postoperativer Frühund Spätinfekte folgt den anerkannten Regeln der septischen Weichteil- und Knochenchirurgie. Frühe Diagnose sowie rasches und situationsadaptiertes Débridement mit adäquater knöcherner Stabilisierung beeinflussen die Langzeitprognose entscheidend.

\section{Schlüsselwörter}

Proximales Femur · Fraktur · Osteosynthesen Fehlschläge · Therapie
Implantatassoziierte Probleme durch direktes Implantatversagen sind selten (Abb. 1). Häufiger sind operationstechnische Fehler und biomechanische Fehleinschätzungen (Abb. 2) sowie patientenbezogene Probleme, wie mangelnde Compliance usw.. Frühfolgen sind Instabilitäten und Dislokationen im Frakturbereich sowie Störungen der Wundund Knochenheilung, die u. U. durch oberflächliche oder gar tiefe Infekte belastet sind. Als Spätfolgen sind Pseudarthrosen, Deformitäten und chronische Osteitiden zu fürchten.

\section{Frakturinstabilität}

Durch gründliche Analyse der Frakturform und verantwortungsbewusste Operationsplanung mit entsprechender Implantatwahl lassen sich unliebsame intra- und postoperative Überraschungen meist vermeiden. Manche Fraktursituationen demaskieren sich indessen erst intraoperativ. Aber auch in dieser Phase bieten sich diverse Möglichkeiten, einer Frakturinstabilität bzw. -dislokation vorzubeugen. Im Bereich von Hüftkopf und Schenkelhals kann drohenden Torsionsinstabilitäten durch die Verwendung so genannter „Antirotationsschrauben “ bei der dynamischen Hüftschraube (DHS) oder den so genannten Miss-a-nailSchrauben bei proximalen Femurnägeln, begegnet werden $[1,6]$.

Die Auswanderung des Trochantermajor-Massivs nach lateral lässt sich bei der DHS-Versorgung mittels Trochanterabstützplatte verhindern. Einem Hochsteigen des Trochanters kann durch zuggurtende Maßnahmen entgegengewirkt werden. Cerclagen aus Draht oder Titanbändern bieten sich an, langstreckige per- und subtrochantere Frakturen zu fesseln, bevorzugt vor der definitiven Osteosynthese mittels DHS oder proxi-

○) Springer-Verlag 2003

Priv.-Doz.Dr. Wolf Strecker Klinik für Unfall-, Handund Wiederherstellungschirurgie, II.Chirurgische Klinik, Klinikum Bamberg, Buger-Straße 80,96049 Bamberg, E-Mail:chirurgie2@klinikum.bamberg.de, Tel.:0951-503-2200, Fax:0951-5032205 


\section{W.Strecker $\cdot$ P. Keppler $\cdot$ M. Schulte}

\section{Therapeutic approaches} after failed operative treatment of proximal femur fractures

\begin{abstract}
Operative treatment of proximal femur fractures is sometimes beset with particular problems. Specific pitfalls and the corresponding etiologies are presented, as are various therapeutic approaches. Implantassociated problems are frequently due to a lack of technical and biomechanical understanding in the surgeon or to inadequate patient compliance. Typical complications are instability and dislocation of the fracture, which cannot be managed except by reconstruction techniques tailored to the specific problems or joint replacement. Disorders of bone healing attributable to osteoporosis, osteopathy, radionecrosis and tumours often require a multidisciplinary therapeutic approach. In selected isolated cases osteogenesis can be improved by electromagenetic stimulation, lithotripsy or low-intensity pulsed ultrasound. Post-traumatic deformities demand thorough analysis of leg geometry, basically by clinical means supplemented by radiography, computed tomography or ultrasound investigation. Indications for the correction of one- and multi-dimensional deformities are given, as are typical examples of correction osteotomies. Acute and chronic infections after ORIF are particularly serious complications. Early diagnosis, prompt and uncompromising debridement and adequate fracture stabilization are of extreme importance, improving long-term outcome decisively.
\end{abstract}

\section{Keywords}

Proximal femur . Fracture $\cdot$ Operative treatment $\cdot$ Pitfalls $\cdot$ Therapeutic solutions
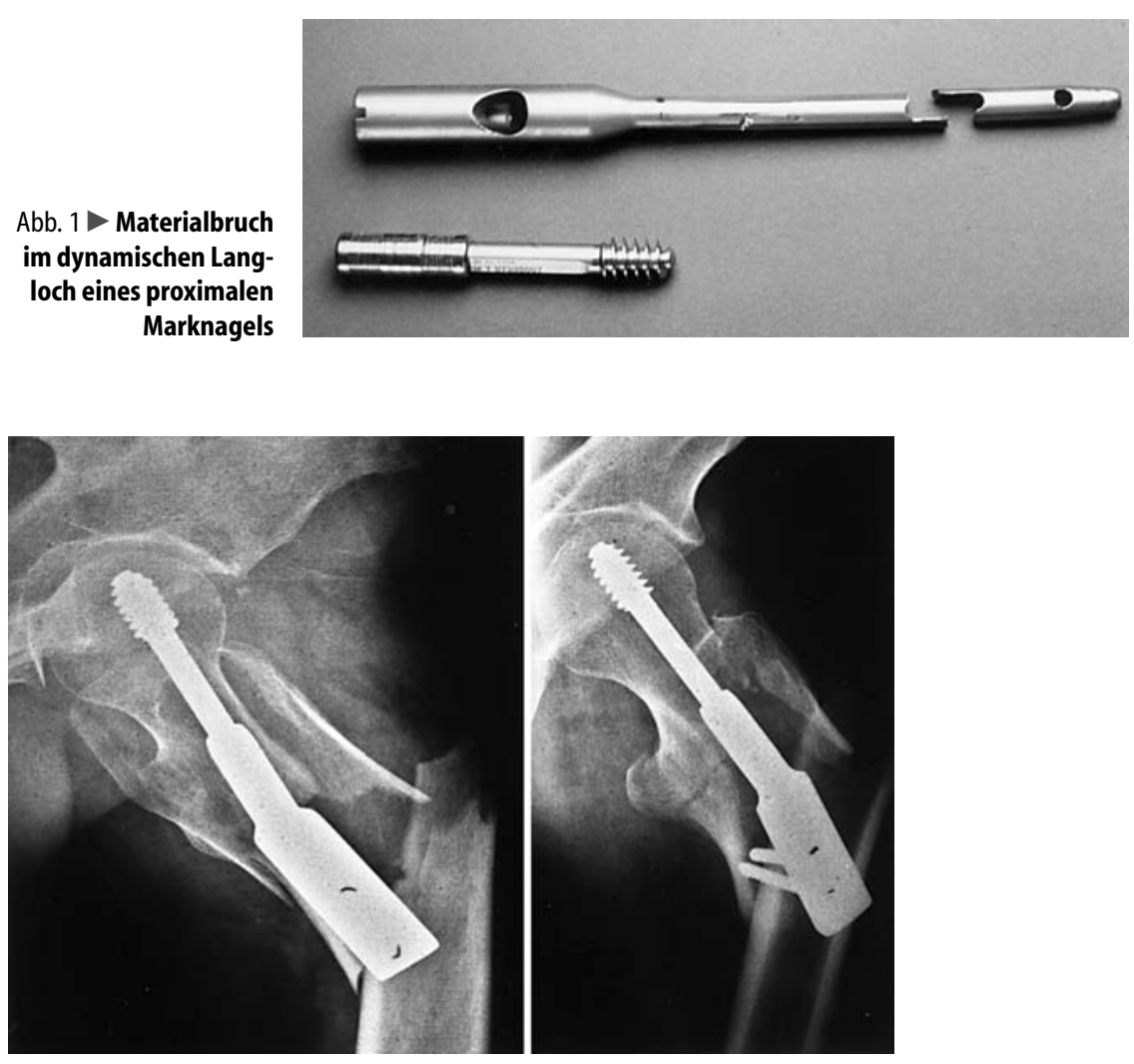

Abb. $2 \Delta$ Ausbruch einer DHS mit 2-Loch-Platte

malem Marknagel. Ebenfalls prophylaktischen Charakter haben initial valgisierende Maßnahmen bei steilen Frakturverläufen entsprechend den Klassifikationen Pauwels II und III [10].

Die laterale Auswanderung dynamischer Hüftschrauben kann im Sinn einer Frakturkompression durchaus erwünscht sein [9], führt aber gelegentlich zu nicht unerheblichen Weichteilirritationen am lateralen Trochanter bzw. am Tuberculum innominatum mit Pseudobursabildung. Hier empfiehlt sich eine lokale Revision, ggf. mit Schraubenwechsel. Ein besonderes Problem ist der so genannte Z-Effekt beim proximalen Femurnagel (PFN): Hier kommt es zum Auswandern der zentralen Schenkelhalsschraube bei gleichzeitigem Einwandern der kürzeren, kranial gelegenen Hüftgleitschraube. Dieses Phänomen ist immer Ausdruck einer Instabilität im Frakturbereich, mutmaßlich bedingt durch Schwingen der Implantatkomponenten.

Ausgeprägtes Wandern der Schrauben mit zunehmender knöcherner Instabilität macht die Revision zwingend. Die Gleit- bzw. Antirotationsschraube sollte hierbei rund $15 \mathrm{~mm}$ kürzer als die
Schenkelhalsschraube gewählt werden. In Einzelfällen kann die zusätzliche Montage einer lateralen Antigleitplatte erwogen werden.

\section{Frakturdislokation}

Frakturdislokationen erfordern generell situationsadaptierte Revisionen in Form einer Reosteosynthese oder einer endoprothetischen Versorgung. Lediglich bei moribunden Patienten sind im Einzelfall ein rein symptomatisches Vorgehen oder die Anlage einer Girdlestone-Situation zu erwägen.

Frakturdislokationen im Bereich von Hüftkopf oder medialem Schenkelhals sind meist von einer gestörten Hüftkopfdurchblutung begleitet. Diese Problematik manifestiert sich frühzeitig durch das so genannte "Cutting-out" von Schenkelhalsschrauben mit gelegentlicher Perforation dieser Schrauben durch den Hüftkopf in das Gelenk. Dementsprechend eingeschränkt sind die Chancen einer knöchernen Heilung. Lediglich bei jüngeren Patienten kann hier ein Erhaltungsversuch, meist als Rekonstruktion mit valgisierender Korrektur, erwogen werden. Ansonsten bleibt in 

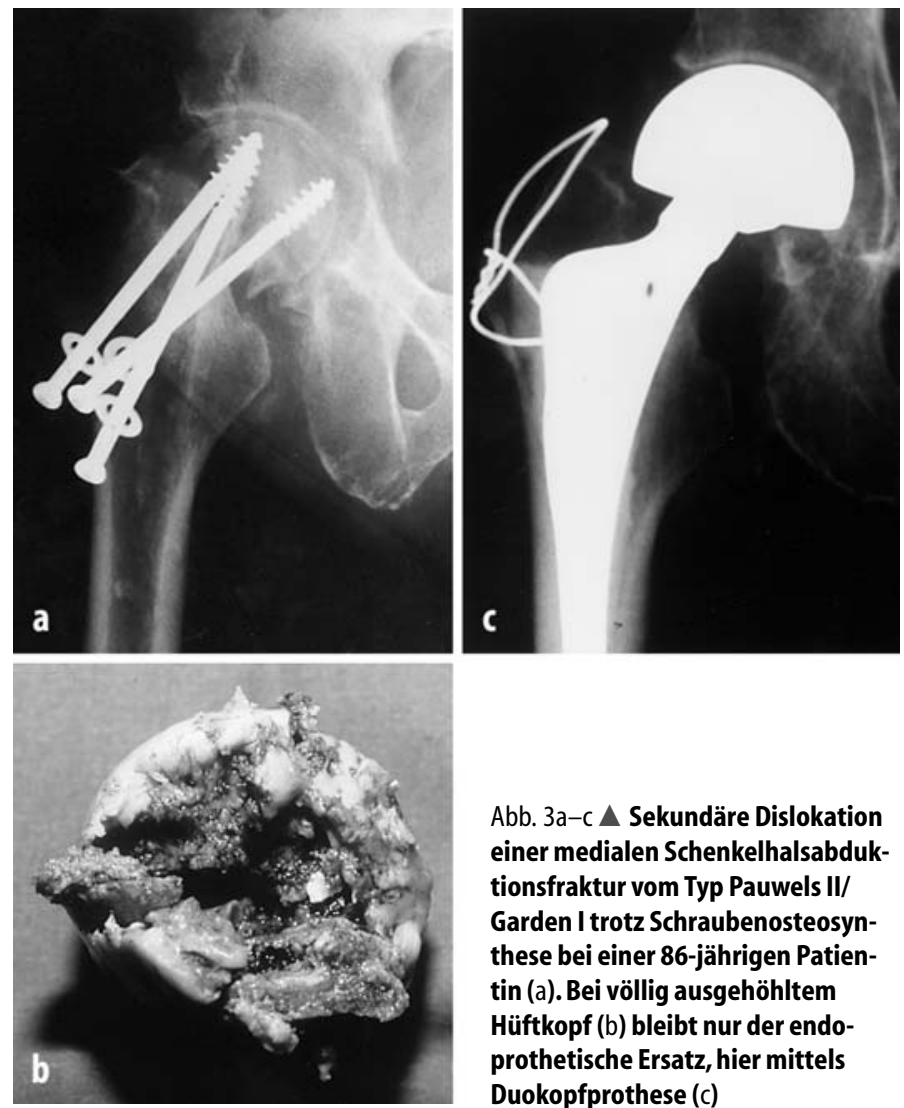

Abb. 3a-c $\Delta$ Sekundäre Dislokation einer medialen Schenkelhalsabduktionsfraktur vom Typ Pauwels II/ Garden I trotz Schraubenosteosynthese bei einer 86-jährigen Patientin (a). Bei völlig ausgehöhltem Hüftkopf (b) bleibt nur der endoprothetische Ersatz, hier mittels Duokopfprothese (c)

derartigen Situationen nur eine differenzierte endoprothetische Versorgung [8] (Abb. 3).

Per- und subtrochantere Frakturdislokationen erfordern im Allgemeinen eine solide Reosteosynthese unter Wahrung biologischer Gesichtspunkte. Verbundosteosynthesen sollten besonderen Problemsituationen vorbehalten bleiben, etwa bei Patienten mit deutlich reduzierter Lebenserwartung.

\section{Knochenheilung}

Die Knochenheilung kann u. a. durch folgende Pathologien gestört sein:

- Osteoporose

- Osteopathie

- Radionekrose

- Tumor

\section{Osteoporose}

Die Rarefizierung der Knochentrabekel macht die Implantatverankerung in Hüftkopf und Schenkelhals zur biomechanischen Herausforderung. Dieses Problem ist auch heute noch nicht in befriedigender Weise gelöst. Antirotations- schrauben, Gleitschrauben und -platten [9], ein drehstabileres Design der Schenkelhalskomponenten [4] und anderes mehr zeugen von dem Bemühen, osteoporosebedingten Instabilitäten mit technischen Mitteln $\mathrm{zu}$ begegnen. Im Fall von Dislokationen muss auf eine der oben dargestellten Behandlungsmethoden ausgewichen werden. Unabhängig von der operativen Frakturbehandlung sind bei sekundärer Osteoporose diagnostische und therapeutische Maßnahmen von internistischer Seite (Endokrinologie, Nephrologie usw.) einzuleiten [14].

\section{Osteopathie}

Osteopathien unterschiedlicher Ätiologie manifestieren sich chirurgisch als knöcherne Deformitäten, als akute traumainduzierte oder schleichende, also pathologische, Fakturen (Abb. 4). Bei Osteopathien erfolgen Diagnostik und Therapie prinzipiell zweigleisig: Während die chirurgische Versorgung den anerkannten Prinzipien der Knochenbruchbehandlung folgt, sollten parallel hierzu angemessene internistische Strategien ausgeschöpft werden. Bereits prä- operativ ist ein entsprechender interdisziplinärer Kontakt wünschenswert, um intraoperativ alle potenziellen diagnostischen Möglichkeiten nutzen zu können. Adäquaten histologischen Untersuchungstechniken kann hier in Einzelfällen eine besonders hohe Aussagekraft zukommen.

\section{Radionekrose}

Tumorrelevante Strahlendosen gehen immer mit einer Beeinträchtigung der lokalen Biologie einher. Die Auswirkungen der Strahlentherapie manifestieren sich oft erst Jahre bis Jahrzehnte post radiatio. Das Frakturrisiko bestrahlter Körperregionen ist dementsprechend erhöht. Zum anderen sind die biologischen Voraussetzungen für eine adäquate Weichteil- und Knochenheilung postoperativ kompromittiert. Gefürchtete Komplikationen sind daher Wundheilungsstörungen, Weichteilnekrosen und -infekte, Pseudarthrosen und Refrakturen (Abb. 5). Bei desolater lokaler Knochenbiologie sind die Möglichkeiten eines Gelenk- und/oder lokalen Knochenersatzes entsprechend den Regeln der Tumorchirurgie zu erwägen [2].

\section{Tumor}

Primäre und sekundäre Knochentumoren demaskieren sich häufig erst durch pathologische Frakturen. Die Ursachen fehlgeschlagener Osteosynthesen begründen sich z. T in der Nichterkennung von Tumoren und in der Nichtbeachtung tumorspezifischer Versorgungsstrategien [2] oder biomechanischer Prinzipien [10]. Nur auf Grundlage einer präzisen Tumordiagnostik mit Staging, einer konzertierten, adjuvanten, multidisziplinären Tumortherapie und der Abschätzung des individuellen Risikoprofils erlaubt letztlich ein auf die individuelle Situation zugeschnittenes Vorgehen. Im Zweifelsfall sind diesbezüglich erfahrene und anerkannte Kollegen $\mathrm{zu}$ konsultieren. Ausgedehnte Ersatzoperationen sind entsprechenden Zentren vorbehalten.

\section{Osteogenese}

Die Osteogenese wird gestört durch

- Durchblutungsstörungen:Rauchen, Diabetes mellitus usw. 


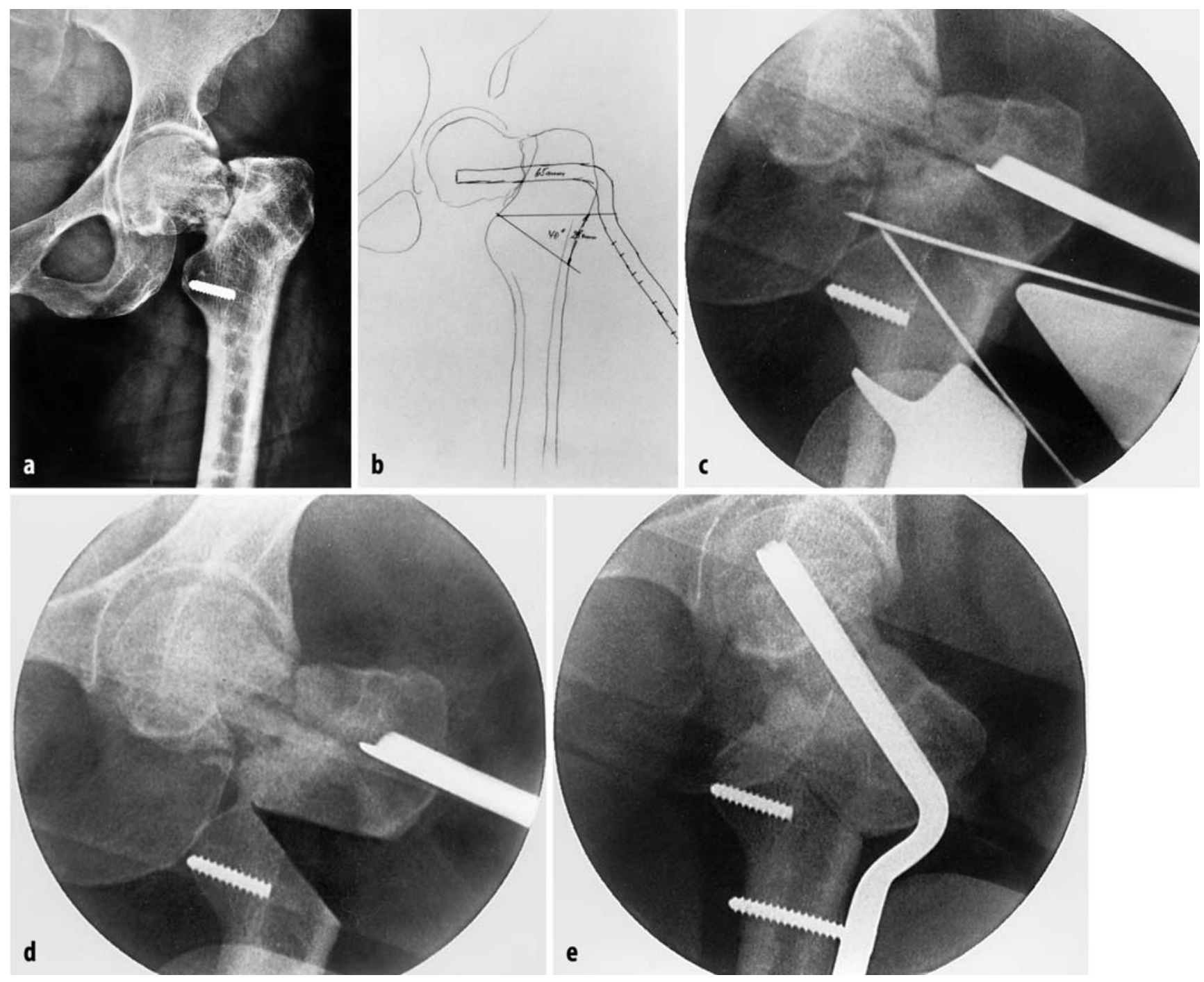

Abb. 4a-e $\Delta$ 40-jährige Patientin mit Charcot-Marie-Tooth-Syndrom und schleichender Refraktur des lateralen Schenkelhalses (a). Planung (b) und operative Umsetzung $(c, d, e)$ der intertrochanteren Valgisierung folgen den biomechanischen Vorgaben von Pauwels [10]

- Medikamente:Kortison, nichtsteroidale Antiphlogistika, Immunsuppressiva usw.

- Inflammation: rheumatoide Arthritis, Immunmangelsyndrome, Sepsis usw.

- Immobilisation

- Radiatio

- metabolische und endokrine Erkrankungen: Diabetes mellitus, Hyperparathyreoidismus usw.

Die Osteogenese wird durch die konsequente Therapie internistischer Pathologien sowie durch eine adäquate Chirurgie positiv beeinflusst. Letztere ist gekennzeichnet durch:

- angemessenes Débridement,

- Infektkontrolle,
- $\quad$ stabile Osteosynthese,

- Spongiosaplastik bzw. Kallusdistraktion und

- $\quad$ suffiziente Weichteildeckung.

Bei gesicherten infektfreien Verhältnissen und Ausschöpfung der oben genannten chirurgischen Therapieoptionen kann bei Pseudarthrosen im Einzelfall eine physikalische Stimulation durch eines der folgenden 3 Verfahren indiziert sein:

- $\quad$ elektromagnetische Stimulation [13]

- Lithotripsie [12]

- gepulster Ultraschall [11]

Die Erfolgsraten einer knöchernen Ausheilung werden mit $70-80 \%$ angegeben. Die zugrunde liegende Pathologie macht allerdings eine direkte Vergleichbarkeit der einzelnen Therapiekonzepte ebenso schwierig wie die Durchführung valider prospektiver Studien.

Die Ergebnisse der magnetfeldinduzierten Wechselstromstimulation sind durchaus viel versprechend [13]. Diese Methode setzt die Implantation eines Elektroüberträgers voraus (Abb. $5 \mathrm{c}, \mathrm{d}$ ). Die Kosten-Nutzen-Relation ist nach der derzeitigen Datenlage als günstig zu betrachten.

Die extrakorporale Stoßwellentherapie zeigt bezüglich der Knochenheilung eher heterogene Ergebnisse [12]. Problematisch sind zum einen die Verfügbarkeit derartiger Einrichtungen, üblicherweise in urologischen Abteilungen angesiedelt, zum anderen die 

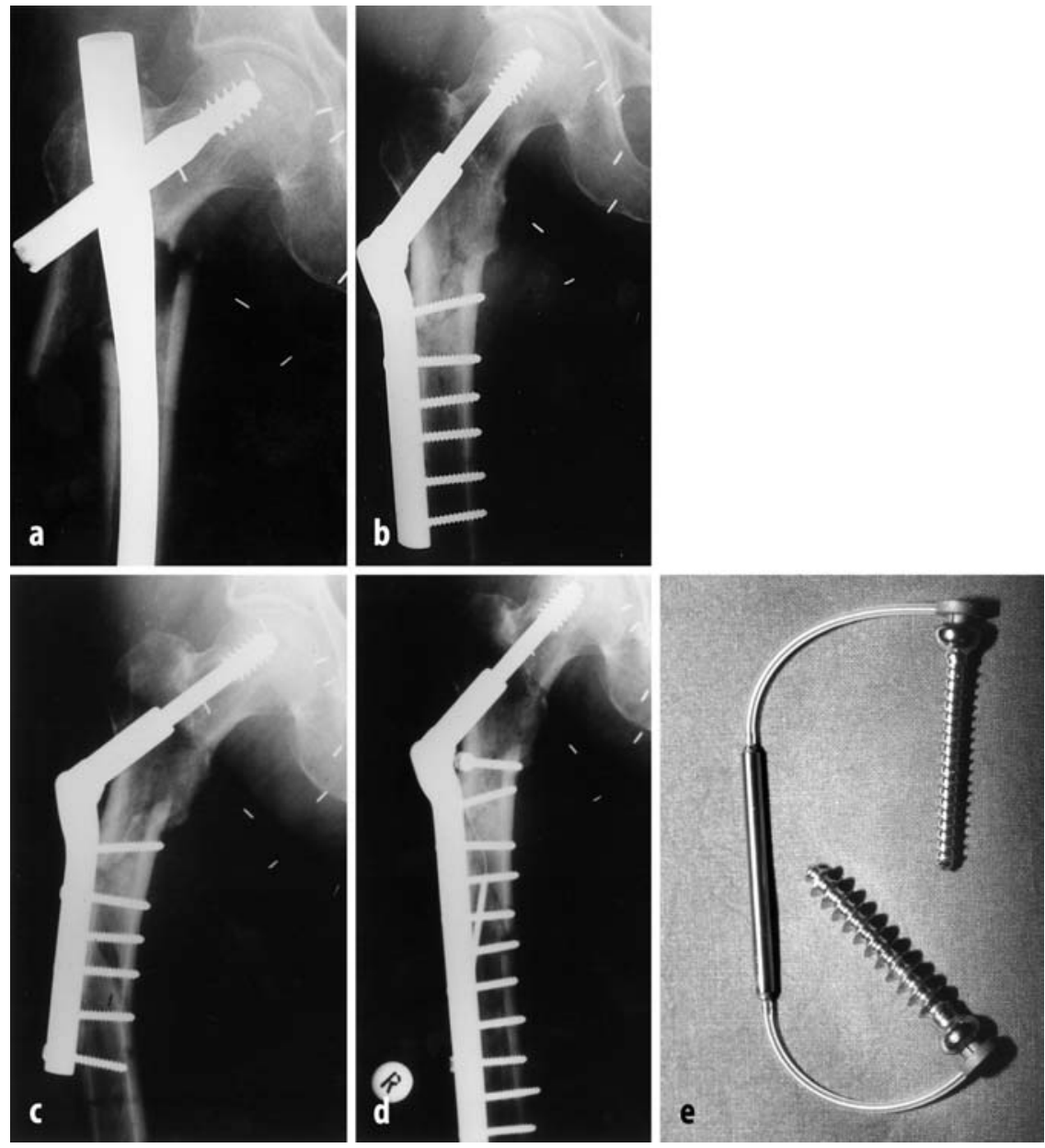

Abb. 5a-e $\Delta$ 65-jährige Patientin mit Pseudarthrose 7 Monate nach Marknagelosteosynthese einer subtrochanteren Femurfraktur. Erhebliche Beeinträchtigung der lokalen Biologie 16 Jahre nach Lymphadenektomie und Radiatio bei Non-Hodgkin-Lymphom (a). Rekonstruktion mit dynamischer Martin-Schraube (DMS) und Anlagerung allogener Spongiosa (b). Neue Fraktur der proximalen Diaphyse im Bereich der histologisch gesicherten Radionekrose (c). Erneute Rekonstruktion mit längerer DMS, Spongiosaplastik und Implantation eines elektromagnetischen Überträgers (d), in e im Detail dargestellt

Schmerzhaftigkeit des Eingriffs selbst. Die Lithotripsie verlangt daher häufig eine flankierende anästhesiologische Betreuung. Derzeit wird diese Behandlungsform von den Krankenkassen nicht unterstützt [12].

Der niederenergetisch gepulste Ultraschall verbindet die Vorteile einer ambulant durchführbaren, schmerzfreien Therapie mit der Unabhängigkeit von Sonderimplantaten [11]. Nachteilig sind die hohen Behandlungskosten sowie die uneinheitliche Anerkennung und Kostenübernahme durch einzelne Krankenkassen.

\section{Deformitäten}

Posttraumatische Deformitäten nach Osteosynthesen proximaler Femurfrak- turen sind wahrscheinlich sehr viel häufiger als allgemein angenommen. Jede 4. Marknagelosteosynthese am Oberschenkel ist von einer Torsionsabweichung $>15^{\circ}$ im Vergleich zur gesunden Gegenseite begleitet [15]. Die Tendenz zu so genannten „biologischen Osteosynthesen" wird offenkundig durch eine höhere Rate posttraumatischer Deformitäten bezahlt.

\section{Indikationen zur Korrekturosteotomie}

Unter Berücksichtigung allgemeiner und lokaler Kriterien der Operabilität erlaubt das Ausmaß der posttraumatischen Deformität eine Indikationsstellung zur Korrekturosteotomie. Die unverletzte Gegenseite dient hierbei als Referenz. Ansonsten orientiert sich die
Achsausrichtung an der Mikulicz-Traglinie bzw. den physiologischen Achsenund Winkelverhältnissen des Beins und ihren jeweiligen Schwankungsbreiten [16]. Für Oberschenkelpaare gelten intraindividuelle Längentoleranzen bis $1,2 \mathrm{~cm}$ und Torsionstoleranzen bis $13^{\circ}$ als physiologisch [16]. Bei posttraumatischen Längendifferenzen sind fixierte Skoliosen der Wirbelsäule auszuschließen.

Zur Prüfung einer Operationsindikation empfiehlt sich ggf. ein probatorischer Längenausgleich durch Schuhsohlenerhöhung für wenigstens 2-3 Monate. Die Indikation zur operativen Torsionskorrektur ist bei fehlendem rotatorischen Nulldurchgang im Hüftgelenk sowohl in Flexion als auch in Extension der Hüfte grundsätzlich gegeben.

Bei mehrdimensionalen Deformitäten relativieren sich die jeweiligen indikatorischen Richtgrößen eindimensionaler Deformitäten.

\section{Korrekturen eindimensionaler Deformitäten}

Ausführliche Angaben zur Korrektur eindimensionaler Deformitäten sind in der Arbeit von Strecker et al. [17] zusammengefasst. Dies betrifft Deformitäten in der frontalen, sagittalen und longitudinalen Achsausrichtung ebenso wie Längen- und Torsionsdifferenzen.

Die Indikation zur Korrektur varischer Deformitäten ohne Pseudarthrose orientiert sich am Beschwerdebild des Patienten, seinen funktionellen Problemen wie Trendelenburg-Hinken und Einschränkungen des koxalen Bewegungsumfangs, an der lateralen trochanteren Ausladung, an der mechanischen Tragachse mit möglichen Fehlbelastun-

Abb. 6a- $\mathrm{h}$ Innentorsionsdeformität von $41^{\circ}$ des rechten Oberschenkels (a) nach proximaler Marknagelosteosynthese (b). Gleichzeitige unfallunabhängige Verkürzung des rechten Beins von $1,4 \mathrm{~cm}$. Daher Indikation zur Außentorsions $\left(40^{\circ}\right)$-Verlängerungs $(1,2 \mathrm{~cm})$-0steotomie subtrochanter mittels Gigli-Säge. Proximal und distal der geplanten Osteotomie dienen die Schanz-Schrauben als Zeiger für die Torsionskorrektur (c). Gigli-Osteotomie (d), Torsionskorrektur um $40^{\circ}$ nach außen (e), gleichzeitige Verlängerung mit Anlagerung von autogener Spongiosa in den Osteotomiespalt und statische Reverriegelung zur Längen- und Torsionssicherung ( $f$ ). Ausheilungsbild 9 Monate nach Korrektur $(\mathrm{g})$ mit regelrechten Torsionsverhältnissen im CT (h) 

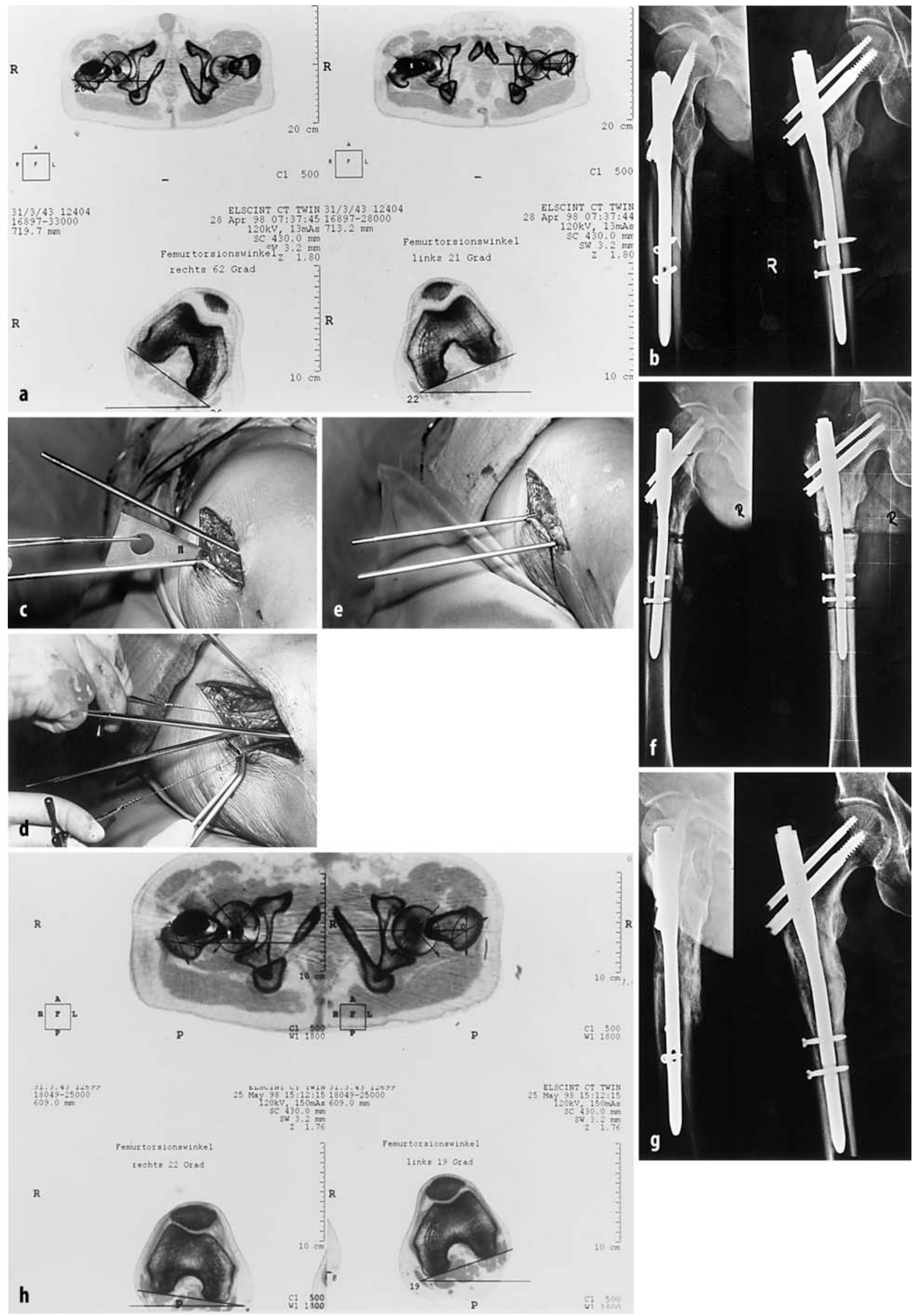
gen von Knie- und Sprunggelenken, am Ausmaß der begleitenden Oberschenkelverkürzung und der koxalen Pfannen-Kopf-Relation. Valgusdeformitäten mit einem zu steilen CCD-Winkel provozieren erhöhte Binnendrücke im Hüftgelenk. Daher besteht bei jüngeren $\mathrm{Pa}$ tienten ggf. die Indikation zur Revarisierung. Damit verbundene Längenverluste sind planerisch und operativ zu berücksichtigen.

Sagittale Deformitäten indizieren lediglich bei Streckdefiziten im Hüftgelenk $>10^{\circ}$ eine Korrekturosteotomie. Die Indikationen zur Korrektur bei longitudinalen Deformitäten sind ebenfalls selten.

Isolierte Längendifferenzen können ab einer Größenordnung von 1,5-2 cm eine Korrekturosteotomie indizieren. Diese Indikation ist allerdings sehr stark vom Alter und dem Allgemeinzustand des Patienten abhängig. Neben einzeitigen und kontinuierlichen Verlängerungen der ursprünglich verletzten Seite sind immer entsprechende Verkürzungsosteotomien der gesunden Gegenseite zu diskutieren.

Torsionskorrekturen erfolgen im Allgemeinen subtrochanter. In Abhängigkeit von Ort und Ursache der Torsionsabweichung können Torsionskorrekturen ausnahmsweise auch intertrochanter indiziert sein. Quere intertrochantere sowie treppenförmige subtrochantere Osteotomien erlauben allerdings lediglich Torsionskorrekturen bis maximal $20^{\circ}$. Schanz-Schrauben von 4 oder $5 \mathrm{~mm}$ Durchmesser, die proximal und distal der geplanten Osteotomie eingedreht werden, empfehlen sich als Winkelzeiger und sichern die Torsionskontrolle (Abb. 6).

Voraussetzung für jede Indikation zur Korrekturosteotomie ist die umfassende Analyse des Problems unter Berücksichtigung patientenspezifischer Eigenheiten. Bereits bei der Entscheidung zu einem operativen Verfahren sollte die Planung einschließlich der intraoperativen Schritte in groben Zügen festliegen. In diese Entscheidung sind konservative und operative Alternativverfahren mit einzubeziehen.

Die Osteotomien erfolgen, in Abhängigkeit vom Apex der Deformität, üblicherweise inter- oder subtrochanter. Lediglich die Drehostoetomien nach Sugioka und Kempf sind am lateralen Schenkelhals lokalisiert. Proximale Fe-

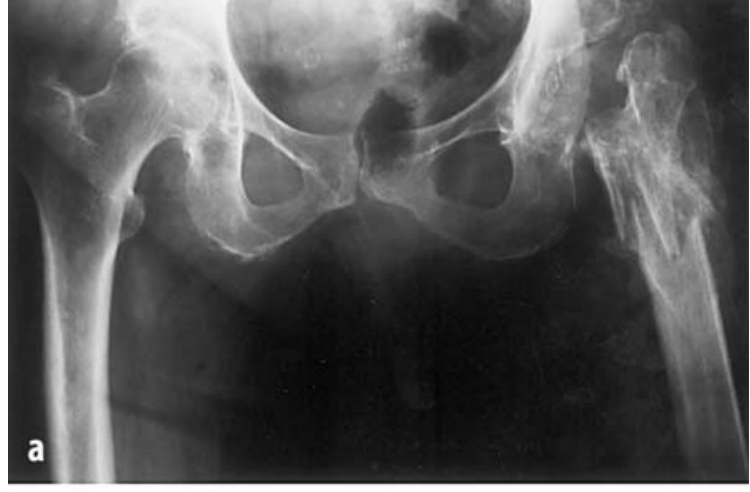

Abb. 7a,b $\Delta$ Girdlestone-Situation 5 Jahre nach Ausbau einer infizierten Hüft-TEP bei einer 82-jährigen Patientin mit Schenkelhalsfraktur und rheumatischer Arthritis (a). Funktionelle Rekonstruktion mit allogener Pfannenbodenplastik, Implantation eines Burch-Schneider-Rings, Einzementieren einer Polyethylenpfanne und eines Revisionsschafts (b)

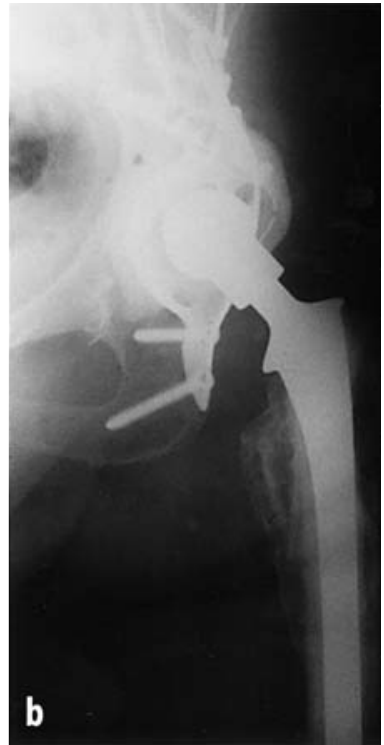

murosteotomien werden nach wie vor bevorzugt mit Winkelplatten stabilisiert. Den weitesten Anwendungsbereich bietet hierbei die $95^{\circ}$-Kondylenplatte. Bei Valgisierungen empfehlen sich $120^{\circ}-$ oder $130^{\circ}$-Winkelplatten, bei Varisierungen gekröpfte Rechtwinkelplatten. Gelegentlich lassen sich posttraumatische Deformitäten elegant über liegende Implantate ausgleichen. Dies bietet sich für Torsions- und Längenkorrekturen nach vorangegangener Osteosynthese mit Marknagel und Schenkelhalsschraube an (Abb. 6). Weitere Einzelheiten sind entsprechenden Übersichten zu entnehmen $[16,17]$.

\section{Infekte}

Die Behandlung von Früh- und Spätinfekten nach Osteosynthesen am proximalen Femur erfolgt nach den anerkannten Regeln der septischen Weichteil- und Knochenchirurgie [7]. Aufgrund der insgesamt guten Durchblutungssituation im Bereich des proximalen Femurs sind Infekte insgesamt relativ selten. Nichtsdestotrotz muss auch hier mit zunehmenden Komplikationsraten aufgrund des höheren Lebensalters und der allgemein wachsenden Komorbidität der Patienten gerechnet werden. Entscheidend sind eine frühe Diagnose und ein rasches Handeln bei ersten Infektzeichen. Hierbei müssen alle Regeln einer so genannten ,guten Chirurgie“, wie oben skizziert, beherzigt werden. Von besonderer Wichtigkeit ist ei- ne rasche und radikale Revision, um einer tieferen Ausbreitung und Chronifizierung von Infekten vorzubeugen. Derartige radikale Revisionen werden durch die modernen Möglichkeiten der Vakuumversiegelung erleichtert [3].

Sollten derartige Maßnahmen nicht zum gewünschten Erfolg führen bzw. zu spät eingeleitet worden sein, bleibt in manchen Fällen nur die radikale Revision mit vollständiger Entfernung des Osteosynthesematerials und einer situationsadaptierten Nekrosektomie. Erst nach sicherer Infektkontrolle können derartige Defektsituationen einer sekundären Rekonstruktion zugeführt werden (Abb. 7). Selbstverständlich sind weitere Infekt begünstigende Ursachen adäquat zu behandeln, bei entsprechenden Konstellationen ggf. mittels eines multidisziplinären Ansatzes.

\section{Literatur}

1. Bonnaire F (2003) DHS - Stärken und Schwächen der Platten-Schrauben-Kombination. Trauma Berufskrankh 5 [Suppl 2]:S162-S170

2. Campanacci M (1999) Bone and soft tissue tumors. Springer, Berlin Heidelberg New York

3. Fleischmann W, Strecker W, Bombelli M, Kinzl L (1993) Vakuumversiegelung zur Behandlung des Weichteilschadens bei offenen Frakturen. Unfallchirurg 96:488-492

4. Friedl W, Vögeli S, Clausen J (2000) Experimentelle Untersuchung zur Bedeutung des Kraftträgerprofiles für die Ausbruchgefährdung bei proximalen Verriegelungsnagelsystemen. Hefte Unfallchirurg 275:470-471 
5. Gonschorek 0 (2003) Komplikationen mit dem proximalen Femurnagel - Vermeidung und Management. Trauma Berufskrankh 5 [Suppl 2]: S171-S174

6. Karich B (2003) Probleme mit dem $\gamma$-Nagel Optimierungsmöglichkeiten. Trauma Berufskrankh 5 [Suppl 2]:S175-S183

7. Kinzl L, Bauer G (1995) Therapie der posttraumatischen Osteitis. Hefte Unfallchirurg 255

8. Neugebauer R (2001) Differenzierte Endoprothetik bei Schenkelhalsfrakturen. Vortrag gehalten auf der Unfallmedizinischen Tagung, Leipzig 2001

9. Olsson O, Ceder L, Hanggard A (2001) Femoral shortening between the Medoff sliding plate and the compression hip screw.J Bone Joint Surg $\mathrm{Br} 83: 572-578$
10. Pauwels F (1973) Atlas zur Biomechanik der gesunden und kranken Hüfte.Springer, Berlin Heidelberg New York

11. Pommer A, Muhr G (2001) Gepulster Ultraschall.Trauma Berufskrankh [Suppl 1] 3:76-79

12. Rompe JD, Rosendahl T, Schöllner C, Riedel C (2001) Lithotripsie bei femoralen oder tibialen Pseudarthrosen. Trauma Berufskrankh [Suppl 1] 3:73-75

13. Schmit-Neuerburg KP (2001) Indikation und klinische Ergebnisse der magnetfeldinduzierten Wechselstromstimulation verzögert heilender Frakturen und Pseudarthrosen. Trauma Berufskrankh [Suppl 1] 3:66-72

14. Schulz W, Deuber HJ (2001) Osteoporose Krankheitsbild und therapeutische Möglichkeiten. Dustri, Deisenhofen
15. Strecker W, Suger G, Kinzl L (1996) Lokale Komplikationen der Marknagelung. Orthopäde 25: 274-291

16. Strecker W, Keppler P, Kinzl L (1997) Posttraumatische Deformitäten - Analyse und Korrektur.Springer, Berlin Heidelberg New York

17. Strecker W, Keppler P, Kinzl L, HehI G (1998) Proximale Femurosteotomien zur Korrektur posttraumatischer Veränderungen. Chirurg 69: 1153-1160

18. Weckbach $A$ (2001) Schenkelhalsfrakturen beim jüngeren Patienten - Problematik und Therapie. Vortrag gehalten auf der Unfallmedizinischen Tagung, Leipzig 2001 